\title{
The high voltage system for the novel MPGD-based photon detectors of COMPASS RICH-1
}

\author{
J. Agarwala ${ }^{a}$, R. Birsa ${ }^{a}$, F. Bradamante ${ }^{a, b}$, A. Bressan ${ }^{a, b}$, C. Chatterjee ${ }^{a, b}, \mathbf{P}$. \\ Ciliberti $^{a, b}$, S. Dalla Torre ${ }^{\star a}$, S. Dasgupta ${ }^{a}$, B. Gobbo ${ }^{a}$, M. Gregori ${ }^{a}$, G. Hamar $^{a}, \mathbf{S}$.

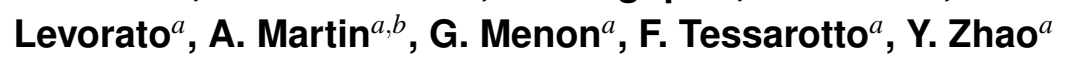 \\ ${ }^{a}$ INFN, Sezione di Trieste, Trieste, Italy \\ ${ }^{b}$ University of Trieste, Trieste, Italy \\ E-mail: Silvia.DallaTorre@ts.infn.it
}

\begin{abstract}
The architecture of the novel MPGD-based photon detectors of COMPASS RICH-1 consists in a large-size hybrid MPGD multilayer layout combining two layers of Thick-GEMs and a bulk resistive MICROMEGAS. Concerning biasing voltage, the Thick-GEMs are segmented in order to reduce the energy released in case of occasional discharges, while the MICROMEGAS anode is segmented in pads individually biased at positive voltage, while the micromesh is grounded. In total, there are ten different electrode types and more than 20000 electrodes supplied by more than $100 \mathrm{HV}$ channels. Commercial power supply units are used. The original elements of the power supply system are the architecture of the voltage distribution net, the compensation, by voltage adjustment, of the effects of pressure and temperature variation affecting the detector gain and a sophisticated control software, which allows to protect the detectors against errors by the operator, to monitor and log voltages and current at $1 \mathrm{~Hz}$ rate and to automatically react to detector misbehaviors.

The HV system and its performance are described in detail as well as the electrical stability of the detector during the operation at COMPASS.
\end{abstract}

5th International Conference on Micro-Pattern Gas Detectors (MPGD2017)

22-26 May, 2017

Philadelphia, USA

\footnotetext{
*Speaker.

$\dagger$ corresponding author
} 


\section{Introduction}

Novel single photon detectors based on MPGD technologies [1] have been developed for the upgrade of the RICH-1 detector [2] of the COMPASS [3] experiment at CERN SPS. They have been installed during the 2015-2016 Winter shut-down of the SPS and operated during the 2016 and 2017 COMPASS data taking.

\section{Requirements}

The requirements for the High Voltage (HV) system originate from the architecture of the novel detectors, consisting in a hybrid MPGD arrangement (Fig. 1): two layers of THick GEMs (THGEM) [4], the first one also acts as a reflective photocathode (its top face is coated with a CsI film), are coupled to a MicroMegas (MM) [5] on a pad segmented anode. The MM is resistive by an original implementation making use of discrete elements: HV is applied to the anode pads, each one protected by an individual resistor, while the signals are collected from a second set of pads, parallel to the first ones, embedded in the anode PCB where the signal is transferred by capacitively coupling (Fig. 2). The detectors are operated with a gas mixture $\mathrm{Ar}: \mathrm{CH}_{4}=50: 50$, selected for optimal extraction of the photoelectrons from the converting CsI film to the gaseous atmosphere [2].

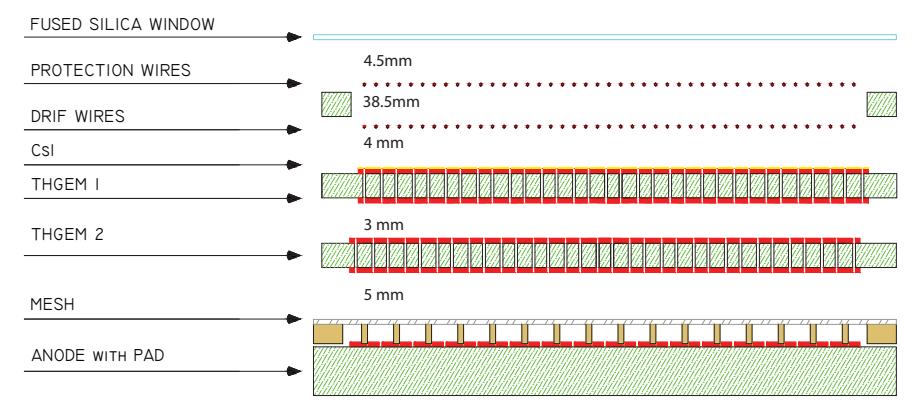

Figure 1: Sketch of the hybrid single photon detector (image not to scale).

For what concerns the active multipliers, namely THGEMs and MM gaps, each $600 \times 600 \mathrm{~mm}^{2}$ detector is formed by two $600 \times 300 \mathrm{~mm}^{2}$ units arranged side by side within a single detector (Fig.s 3,4), while the protection and drift wire planes are in common for the two units.

The typical voltages applied to the detector electrodes are (with reference to electrodes as indicated in Fig. 1): protection wires at $-300 \mathrm{~V}$, drift wires at $-3400 \mathrm{~V}$, THGEM 1, top at $-3200 \mathrm{~V}$, THGEM 1, bottom at -2000 V, THGEM 2, top at -1700 V, THGEM 2, bottom at $-500 \mathrm{~V}$, mesh grounded and anode at $+600 \mathrm{~V}$.

\section{The high voltage distribution scheme and the high voltage system}

The protection and drift wires are powered by a power supply channel each.

The THGEM plates are physically segmented in 12 longitudinal segments with area $24 \times 600 \mathrm{~mm}^{2}$ (Fig. 4). Concerning power supply, six segments of the top (bottom) face, corresponding to an area 


\section{(a)}
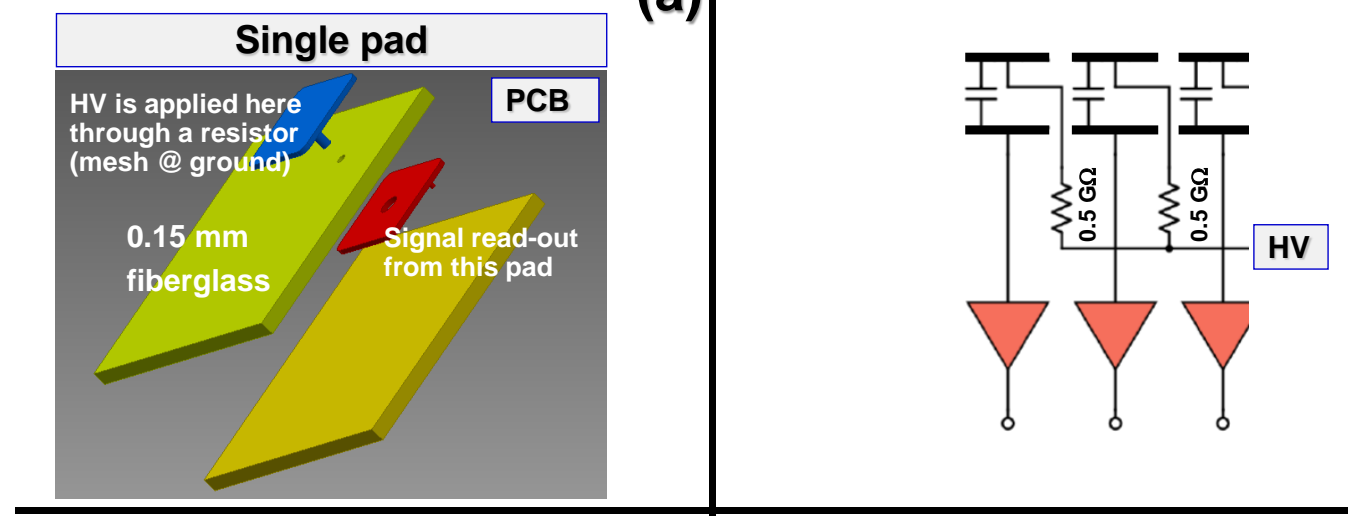

(b)
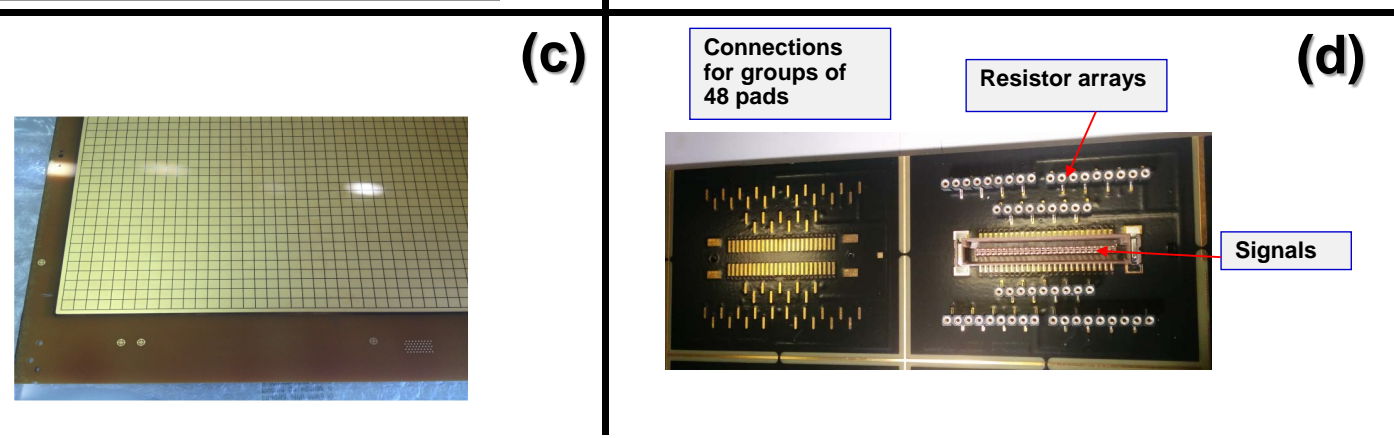

Figure 2: The resistive MM by discrete elements. (a) Sketch illustrating the principle by the exploded view of the PCB layers: the blue pad is the anode electrode of the MM; the red pad is embedded in the PCB and the signal is transferred from the blue to the red pad by capacitive coupling. (b) The principle is illustrated by the electrical scheme: the top elements of the capacitors are the pad forming the MM anode (blue pad in (a)), the bottom elements of the capacitors (red pad in (a)) are connected to the front-end electronics. (c) Picture of the MM anode PCB, front view. (d) Picture of the MM anode PCB, rear view, detail: the connectors serving 48 pads are grouped together; the signal connectors and the resistor arrays connectors can be seen.

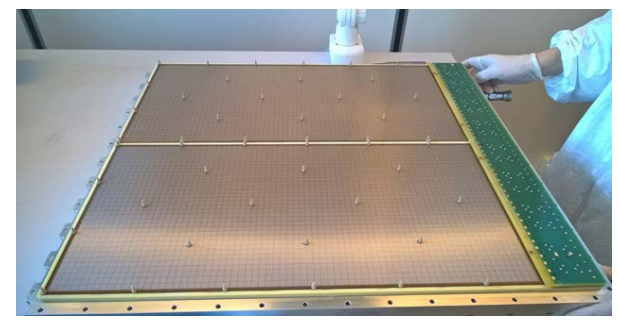

Figure 3: Picture, taken during the detector construction, of two MM units arranged in a detector.

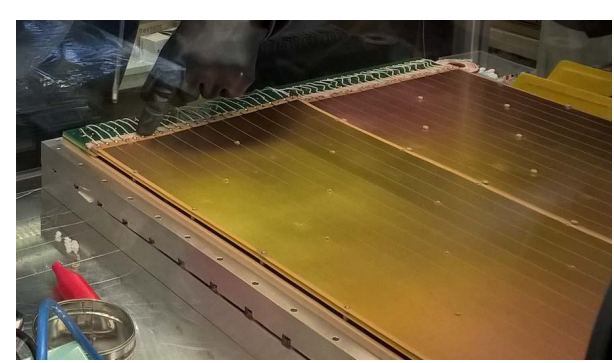

Figure 4: Picture, taken during the detector construction, of two THGEMs arranged in a detector. The 12 physical segments of each THGEM are visible. 
of $180 \times 600 \mathrm{~mm}^{2}$ referred to as a "sector" in the following, are powered by a single individual supply channel, where the voltage is distributed to the individual segments according to the scheme shown in Fig. 5. The resistors and fast diodes in series with each segment aim at suppressing the cross-talk of the voltage swing when a discharge affects one of the segments.

The distribution scheme of the power to the MM pads is shown in Fig. 2 (b) and two power supply channels per unit are used, each one supplying 2450 pads; this area corresponds to that of a sector: from the power supply point of view, each detector, formed by two units, is powered by sectors, two per unit and, therefore, four per chamber.

The preservation of the correct electric field configuration at the lateral detector edges, where the detector frames are very near to the active elements in order to minimize the dead area between adjacent detectors, imposes to preserve the field shape by the presence of two auxiliary electrodes at the two lateral sides of the detector frames, corresponding to further four supply channels per detector.

In total, the supply system for the four detector set includes more than 100 channels. Commercial power supply units by CAEN ${ }^{1}$ are used. THGEMs are powered by A1561HDN 12 channel units, capable of voltage supply up to $-6 \mathrm{kV}$ and current monitor with $50 \mathrm{pA}$ resolution, fully satisfactory for our application; MMs are supplied by A7030DP 12-channel units, with maximum voltage of $+3 \mathrm{kV}$ and $2 \mathrm{nA}$ resolution of the current monitor; this resolution is marginal with respect to our needs and the off-set of the current monitor is unstable. The power supply units are housed in two type SY4527 main frames, one placed near the top detectors and one placed near the bottom ones to minimize the HV cable, which is $5 \mathrm{~m}$.

HV channel setting within a detector is highly correlated, making the correct manual operation a complex and risky task. Therefore, a custom-made control system has been realized; it is written in C++ making use of the wxWidgets library and designed so to comply with the COMPASS experiment slow control system. The reference HV values are accompanied by individual "OwnScale" of each channel to make fine-tuning for gain uniformity easier. An autodecrease HV algorithm is implemented to protect the detectors in case of a too high current spark rate. The voltage and current monitored by the power supply are logged at $1 \mathrm{~Hz}$ rate. The voltages are continuously corrected on the base of the measured environmental parameters, namely temperature and pressure, in order to preserve the detector gain stability. The correction algorithm is the result of dedicated laboratory tuning exercises:

$$
V(P, T)=V_{0}\left(1+0.5\left(P / P_{0} \cdot T_{0} / T-1\right)\right)
$$

where $\mathrm{V}$ is the voltage, $\mathrm{P}$ is the absolute pressure in mbar, $\mathrm{T}$ is the temperature in degrees Kelvin and $\mathrm{V}_{0}, \mathrm{~T}_{0}$ and $\mathrm{P}_{0}$ refer to reference conditions.

The control system is completed by a GUI interface for user access.

\section{The electrical performance of the the novel MPGD-based photon detectors of COMPASS RICH-1}

No HV-level trip has been observed at the supply channels over about seven months of operation, thanks to the schemes adopted for the HV distribution. Current sparks are present, corre-

\footnotetext{
${ }^{1}$ CAEN S.p.A., Via della Vetraia, 11, 55049 Viareggio, Lucca, Italy
} 


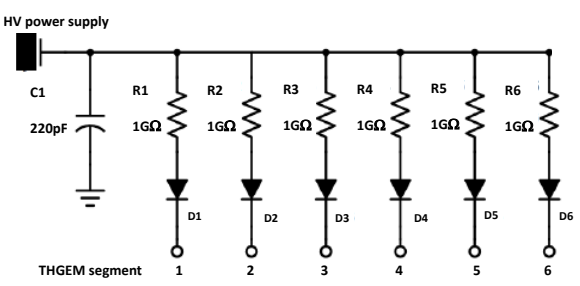

Figure 5: Scheme of the voltage distribution to six THGEM segments of the top (bottom) face.

sponding to temporary discharges. The stability of the voltage from the supply guarantees that only a portion of the detector is affected, namely, a physical segment in case of THGEMs and a pad area in case of MMs. The recovery time, monitored via the current information, is $\lesssim 10 \mathrm{~s}$ for current sparks in THGEM and about 1-2 s in MMs. Sparks in the two THGEMs are 100\% correlated. All the observed sparks in MMs are accompanied by sparks in THGEMs and not vice versa, suggesting that they are induced by misbehaviors in THGEMs.

Sparks rates, typically not exceeding $20 / \mathrm{h}$ in total for the four novel detectors, do not exhibit a remarkable dependence on the beam intensity (Fig. 6). Sparks correlated in time have been observed for THGEM segments, also when they are not contiguous. These observations suggest that a major source of the observed sparks are cosmic rays that, occasionally, can produce relevant ionization in these detectors which are oriented almost vertically.

Three sectors have exhibited weaker electrical stability during the first year of operation of the novel detector, corresponding to almost $20 \%$ of the active surface. The observations about the current spark correlations suggest that they are related to THGEM regions with weaker performance. Therefore, a dedicated measurement campaign was performed in the 2016-2017 Winter shut-down of the SPS, to identify, within each single sector, the weak segments in order to supply them separately and move the weak performance to smaller areas: a segment represent only $1 \%$ of the total active area. THGEM 1 and THGEM2 of each detector have been studied separately. Each segment of the THGEM under study was separately powered at increasing voltages, in a sequence of 10-minute steps. The spark-rate was measured, segment by segment during the step and the voltage was decreased, instead of increasing it, if the number of sparks was exceeding 5. The voltage and current parameters were logged and the exercise extended for a few consecutive days for each THGEM. The total time that a segment could stand a given voltage, the number of sparks versus voltage and the spark rate at given voltage were used to monitor the segment quality and they have provided consistent indications, from which the weak segments have been identified. A single weak segment was identified in the THGEMs used as THGEM 1, in spite of the presence of the CsI coating and, more in general, these THGEMs could stand higher voltage than the THGEMs used as THGEM 2. This unexpected outcome can be related to the extra preparatory processes required to use a THGEM as photocathode substrate: Ni-Au coating and one more cleaning step. In total 10 weak segments were identified and separately powered, corresponding to $10 \%$ of the total active surface.

A few local shorts have developed over months of operation in the MM gaps. They affect single, isolated pads, they are observed through an increase of the supply current and could not be 
removed by the application of reversed bias. We do not know the cause. After seven months of operation the observed rate is 1 per mil.

The detector gain has been observed over periods of months by analyzing the collected data. The gain is extracted from the amplitude spectrum of the signals generated by the single photoelectrons; it is stable at about the 5\% level apart in a single sector, for which further investigation is ongoing.

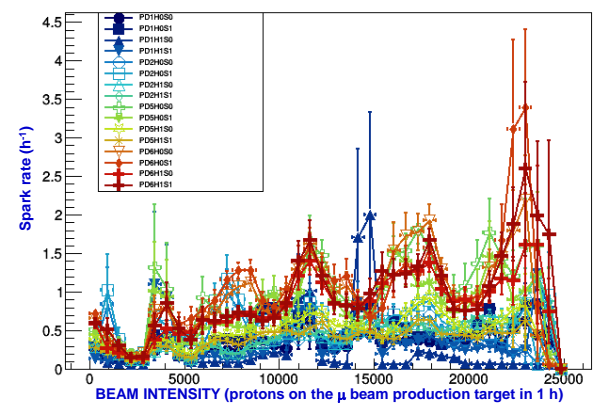

Figure 6: Spark rate versus beam intensity for the 16 sectors of the four chambers.

\section{Conclusions}

In spite of the complexity of hybrid MPGDs, the HV system with sophisticated control, that we have implemented, guarantees safety operation, the collection of the information required for understanding and monitoring the detector behavior and their satisfactory electrical stability at gains around $15 \mathrm{k}$, a non-trivial achievement considering that, so far, all MPGDs operate in experiment at gain below $10 \mathrm{k}$.

\section{ACKNOWLEDGMENT}

The activity is partially supported by the H2020 project AIDA2020 GA no. 654168 .

\section{References}

[1] F. Tessatotto et al., "The novel photon detectors based on MPGD technologies for the upgrade of COMPASS RICH-1", these proceedings and references therein.

[2] E. Albrecht et al., Nucl. Instr. and Meth. A 553 (2005) 215; P. Abbon et al. , Nucl. Instr. and Meth. A 587 (2008) 371; P. Abbon et al. , Nucl. Instr. and Meth. A 616 (2010) 21; P. Abbon et al. , Nucl. Instr. and Meth. A 631 (2011) 26.

[3] P. Abbon et al. , Nucl. Instr. and Meth. A 577 (2007) 455; P. Abbon et al. , Nucl. Instr. and Meth. A 779 (2015) 69.

[4] L. Periale et al., Nucl. Instr. and Meth. A 478 (2002) 377; P. Jeanneret, PhD thesis, Neuchatel University, 2001; P.S. Barbeau et al, IEEE NS-50 (2003) 1285; R. Chechik et al, Nucl. Instr. and Meth. A 535 (2004) 303.

[5] Y. Giomataris et al., Nucl. Instr. and Meth. A 376 (1996) 29.

[6] H. Hoedlmoser et al., Nucl. Instr. and Meth. A 574 (2007) 28. 